\title{
Plasmodium vivax thrombospondin related adhesion protein: immunogenicity and protective efficacy in rodents and Aotus monkeys
}

\author{
Angélica Castellanos, Myriam Arévalo-Herrera/ ${ }^{*}$, Nora Restrepo, Leonel Gulloso ${ }^{* *}$, \\ Giampietro Corradin ${ }^{* * *}$, Sócrates Herrera/ $/{ }^{*}$
}

Instituto de Inmunología del Valle, Universidad del Valle, AA 25574 Cali, Colombia *Malaria Vaccine Drug and Development Center, Cali, Colombia ${ }^{* *}$ Grupo de Investigación en Salud Pública, Universidad San Martin, Cali, Colombia **** Institute of Biochemistry,

University of Lausanne, Epalinges, Switzerland

The thrombospondin related adhesion protein (TRAP) is a malaria pre-erythrocytic antigen currently pursued as malaria vaccine candidate to Plasmodium falciparum. In this study, a long synthetic peptide (LSP) representing a P. vivax TRAP fragment involved in hepatocyte invasion was formulated in both Freund and Montanide ISA 720 adjutants and administered by IM and subcutaneous routes to BALB/c mice and Aotus monkeys. We measured specific humoral immune responses in both animal species and performed a sporozoite challenge in Aotus monkeys to assess the protective efficacy of the vaccine. After immunization both mice and Aotus seroconverted as shown by ELISA, and the specific anti-peptide antibodies cross reacted with the parasite in IFAT assays. Only two out of six immunized animals became infected after P. vivax sporozoite challenge as compared with four out of six animals from the control group. These results suggest that this TRAP fragment has protective potential against P. vivax malaria and deserves further studies as vaccine candidate.

Key words: Plasmodium vivax - malaria vaccine - thrombospondin-related adhesion protein

Thrombospondin related adhesion protein (TRAP) or sporozoite surface protein 2 (SSP2/TRAP) is an antigen from the pre-erythrocyte stage of the malaria parasite Plasmodium. The protein has been found present in the sporozoite micronemes and appears to be released to the parasite surface immediately before the invasion process. It is a transmembrane protein that belongs to the TRAP/Micronemal protein 2 (TRAP/MIC2) family required for sporozoite gliding motility and together with the circumsporozoite protein (CS) it has been found to be essential to the process of malaria sporozoite infection to the hepatocyte (Muller 1993, Sinnis \& Sim 1997). This protein highly conserved among Plasmodium species contains, towards the N-terminus of the protein in $P$. vivax, a conserved fragment (WTPCSVTCGVGVRVRRR) denominated region II-plus (Sinnis \& Sim 1997) located at positions (307-324) which bind to sulfated glycoconjugates (Templeton \& Kaslow 1997). Like the malaria CS protein, SSP2/TRAP binds to the heparan sulfate molecules of the basolateral domain of hepatocytes during the initial contact between hepatocytes and the invading sporozoites (Frevert et al. 1993, Yuda \& Ishino 2004).

Financial support: US National Institute of Allergy and Infections Diseases NIAID/TMRC contract \# AI49486-02, the Instituto Colombiano para el Desarrollo de la Ciencia y la Tecnología, Francisco José de Caldas - Colciencias, contract 142-2000

${ }^{+}$Corresponding author: sherrera@inmuno.org

Received 19 March 2007

Accepted 24 April 2007
Several studies using functional in vitro assays and animals models such as mice and non-human primates have demonstrated that TRAP can block the hepatocytes invasion by sporozoites and therefore confers partial protection against $P$. yoelii, $P$. cynomolgi, and $P$. falciparum infection (Hoffman et al. 1996).

In addition, it has been observed that both, humans vaccinated with $P$. falciparum irradiated sporozoites or individuals naturally exposed to malaria induce $\mathrm{CD}^{+} \mathrm{T}$ cell-dependent cytolytic activity mediated by a HLArestricted epitope derived from SSP2/TRAP, indicating its potential as vaccine candidate (Aidoo et al. 1995, Wizel et al 1995). However, there are no published studies on the immunogenicity or protective efficacy of the $P$. vivax TRAP. Here we describe a pilot immunogenicity study conducted in $\mathrm{BALB} / \mathrm{c}$ mice using the $P$. vivax TRAP derived long synthetic peptide (LSP) which contains the motif for sporozoites and hepatocytes interaction, followed by a pre-clinical study in Aotus monkeys to assess its protective potential.

\section{MATERIALS AND METHODS}

Peptide - We synthesized a peptide corresponding to the amino-acid sequence 209-256 of Salvador I strain $P$. vivax TRAP (PvTRAP) located towards the N-terminus of the protein, which contains the region II motif involved in binding parasites in hepatocytes (Templeton \& Kaslow 1997) (see Fig. 1). This peptide was synthesized under good laboratory practices (GLP) conditions using F-moc chemistry. The peptide contains 6 cysteine residues which were oxidized according to Corradin et al. (2004) and then it was purified by high-performance liquid chromatography (HPLC) to purity higher than $80 \%$ (Sigma Genosys, St Louis, Mo). 


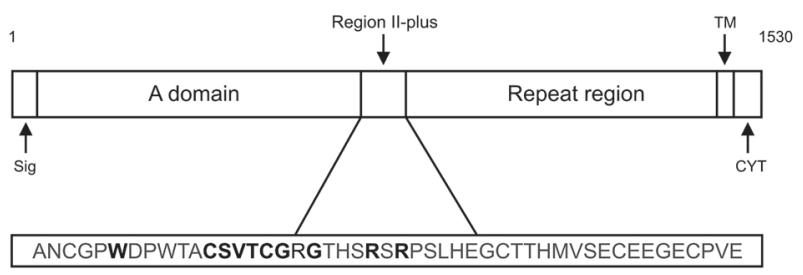

Fig. 1: schematic representation of Plasmodium vivax TRAP protein showing the localization of the synthetic peptide used in the present study. Sig: signal sequence; TM: transmembrane sequence; CYT: cytoplasmic tail. Bold characters represent the cell adhesive motif

Animals - BALB/c mice and Aotus lemurinus griseimembra monkeys from the animal facility of the Malaria Vaccine and Drug Development Center (MVDC) Cali, Colombia, were used. Aotus monkeys were adult male and non-pregnant females, malaria naïve, with body weight above $800 \mathrm{~g}$. Animals were overseen by a veterinarian and were handled under US National Institutes of Health (NIH) Guide for Care and Use of Laboratory Animals. The animal testing protocol was submitted to and approved by the Animal Ethics Committee of the Universidad del Valle.

Immunization schedules - For the pilot study, eight $\mathrm{BALB} / \mathrm{c}$ mice were separated in two groups of four animals each: the experimental group was immunized on days 0,30 , and 60 by subcutaneous (SC) route with 30 $\mu \mathrm{g}$ of PvTRAP formulated in Freund's adjuvants at a 1:1 proportion. The control group was immunized with phosphate buffer saline (PBS) formulated in the same adjuvants following similar immunization schedule. The first vaccination dose was formulated in complete Freund adjuvant (CFA), whereas the other two doses were formulated in incomplete Freund adjuvant. Monkeys were randomly assigned to two groups of six animals per group. The experimental group received IM injections of $100 \mu \mathrm{g}$ PvTRAP peptide formulated in Montanide ISA 720 (SEPPIC, Paris, France) in a proportion of 3:7 antigen:adjuvant as recommended by the manufacturer. Injections were given on days 0,30 , and 90 and a fourth vaccination dose with the PVTRAP peptide formulated in CFA was given on day 150 to determine the potential boosting effect. This latter vaccination was administered subcutaneously, with the LSP formulated in a 1:1 proportion in a mixture (50:50) of CFA and incomplete Freund's adjuvants. The six animals in the control group followed the same immunization schedule as the experimental group but with adjuvant emulsified in PBS without peptides.

Antibody determination - Enzyme-linked immunosorbent assay (ELISA) was done to determine antibody titers to the LSP, using sera from experimental and control animals, collected on the day of each immunization as described previously (Arévalo-Herrera et al. 1998). Briefly, ELISA well plates (Nunc-Immuno Plate, Maxisorp, Roskilde, Denmark) were coated overnight with the LSP $(1 \mu \mathrm{g} / \mathrm{ml})$ in PBS pH 7.2 at $4^{\circ} \mathrm{C}$. After blocking with $5 \%$ BSA in PBS, serial dilutions of test serum were added and incubated for $1 \mathrm{~h}$ at room temperature.
Then, antibodies were detected using phosphatase-conjugated anti-mouse IgG (Sigma Chemical Co., St Louis, MO) in case of rodent and with phosphatase-conjugated anti-human IgG for monkeys (Sigma), and the plates were developed using an enzymatic reaction with paranitrophenyl phosphate substrate. The results were expressed as the reciprocal of the endpoint of the last dilution at which $\mathrm{OD}_{450}$ for control serum (pre-immunization serum) was greater than the average $\left(\mathrm{OD}_{450}\right)$ plus three standard deviations (SD).

Native protein recognition - We performed indirect immunofluorescence assays (IFAT) to assess the recognition of the $P$. vivax native TRAP by specific antipeptide antibodies, IFAT multi-well slides were prepared using $10 \mu \mathrm{l}$ of a parasite suspension containing $2 \times 10^{3}$ $P$. vivax sporozoites obtained from experimentally infected Anopheles albimanus as described before (Herrera et al. 1997). Slides were stored frozen at $-70^{\circ} \mathrm{C}$ until use. For IFAT the slides were thawed and dried at room temperature and then incubated with double serial dilutions of antibodies from experimental and control mice and monkeys for $1 \mathrm{~h}$. Three washes were done, and reaction was developed with fluorescein-conjugated goat antimouse $\mathrm{IgG}(\mathrm{H}+\mathrm{L})$ in the case of rodent sera, and with fluorescein-conjugated goat antihuman $\operatorname{IgG}(\mathrm{H}+\mathrm{L})$ (Jackson Immunoresearch Laboratories, Inc., Baltimore, MD) diluted at 1:100 for the monkey studies. The slides were examined by epifluorescence microscope. Antibody titers were expressed as the positive end point dilution that showed specific fluorescence of the sporozoite surface.

Aotus IFN- $\gamma$ ELISPOT - To determine IFN- $\gamma$ production by specific mononuclear cells induced by synthetic peptide, ELISPOT was carried out using a human IFN- $\gamma$ kit (Mabtech, Stockholm, Sweden) as described elsewhere (Herrera et al. 1997). Peripheral blood mononuclear cells (PBMCs) were obtained from whole blood of experimental and control monkeys on days 0, 30, 90, and 150 by Ficoll-Histopaque density gradient. A total of $2 \times 10^{5}$ cells per well were cultured in plates (Millipore, MAHA S45, Bedford, MA, US) for $40 \mathrm{~h}$ at $37^{\circ} \mathrm{C}$ and $5 \% \mathrm{CO}_{2}$ in the presence of $10 \mu \mathrm{g} / \mathrm{ml}$ of PvTRAP peptide. The plates were pre-coated overnight with mouse antihuman IFN- $\gamma$ monoclonal antibodies (1-DIK Mabtech) blocked with Roswell Park Memorial Institute (RPMI) containing $10 \%$ fetal calf serum for $2 \mathrm{~h}$ at $37{ }^{\circ} \mathrm{C}$ and $5 \% \mathrm{CO}_{2}$. Plates were washed and treated with biotinylated goat antihuman IFN- $\gamma$ polyclonal antibody followed by color development using the reagents supplied in the kit. IFN- $\gamma$ cell production was determined by comparison with positive controls stimulated with phytohemaglutinine (PHA) and expressed as $10^{6}$ spotforming units (SFU).

Protective efficacy of vaccination - We attempted to assess the efficacy of vaccination to prevent infection after challenge with viable $P$. vivax sporozoites, using prepatent period, parasitemia levels and duration of infection and anemia as markers of protection. Aotus 
were intravenously inoculated with $2 \times 10^{4} P$. vivax sporozoites obtained by salivary gland dissection of $A$. albimanus mosquitoes, previously fed with blood from a human patient as previously described (Jordan et al. 2005). Infection was performed on day 60 after last immunization (day 210). Giemsa stained thick and thin blood smears (WHO 1991) were done before challenge and every other day from day 15 after challenge to detect parasitemia. We used the thin smear to determine the percentage of infected red blood cells (RBC) contained in a total of $1000 \mathrm{RBC}$, whereas thick smears were used to score the number of parasites in 300 leucocytes. We defined presence of one parasite as positive and marking the end of the prepatent period. Each slide was read independently by two microscopists in a blinded manner for group and sample day. To confirm the results, all samples that were negative by thin and thick smears were tested by a nested polymerase chain reaction (PCR) on Whatman filter paper. Filter paper was soaked in $100 \%$ methanol for $45 \mathrm{~min}$, then methanol was removed and DNA was precipitated from methanol with $50 \mu \mathrm{l}$ of water and used to perform a species-specific nested PCR as previously described (Snounou et al 1993). In order to determine the potential development of anemia, all monkeys were subjected to hematocrit and hemoglobin determination every other day on the same days as parasitemia.

Statistical analysis - Protective efficacy was evaluated by comparing the incidence of infection and survival time between the experimental and control groups and differences were determined using square chi test. Incidence of infection was defined as the number of positive cases over the number of animals exposed to infection. In addition, proportion of animals without infection for each group was defined as the time from inoculation of sporozoites to the development of infection detected by PCR. Estimated proportion curves were calculated using the Kaplan Meier method (Berry et al. 1992). Differences between experimental and control groups were analyzed by a log rank test conducted at the two-sided, 0.05 level of significance.

\section{RESULTS}

Immunogenicity of PvTRAP peptide - All BALB/c mice seroconverted after the first immunization dose as determined by ELISA. Titers of anti-peptide antibodies increased steadily after the first immunization to reach titers ranging from 1 to $128 \times 10^{3}$ after the third injection (Table I). Similarly, sera of two of the four mice vaccinated with the TRAP LSP were able to recognize sporozoites in IFAT assays. Although all Aotus monkeys also seroconverted after the complete immunization regime, antibody levels that ranged from $1 \times 10^{2}$ to $1 \times 10^{4}$ were significantly lower than those of mice. Most animals needed at least two immunization doses of PvTRAP peptide formulated in Montanide ISA 720 adjuvant to produce detectable antibody levels, and four of the six monkeys produced a rapid decrease of specific antibody titer after the third immunization to undetectable levels
TABLE I

Titers of anti-PvTRAP antibodies developed in BALB/c mice

\begin{tabular}{lcccrrrrr}
\hline & \multicolumn{3}{c}{ ELISA $^{a}$} & \multicolumn{4}{c}{ IFAT $^{b}$} \\
\hline Mouse/day & 0 & 30 & 60 & 90 & 0 & 30 & 60 & 90 \\
\hline E1 & 0 & 2 & 16 & 64 & 0 & 100 & 400 & 400 \\
E2 & 0 & 2 & 2 & 1 & 0 & 0 & 0 & 0 \\
E3 & 0 & 2 & 16 & 16 & 0 & 100 & 200 & 400 \\
E4 & 0 & 2 & 64 & 128 & 0 & 0 & 0 & 0
\end{tabular}

$a$ : Elisa anti-PvTRAP antibodies titer expressed as the reciprocal of the last positive dilution $\times 10^{3} ; b$ : IFAT performed with air-dried $P$. vivax sporozoites. Titers represent the reciprocal of the serum end-point dilution.

TABLE II

Titers of anti-PvTRAP peptide antibodies developed in of Aotus monkey

\begin{tabular}{|c|c|c|c|c|c|c|c|}
\hline \multirow[b]{2}{*}{ Mouse/day } & \multicolumn{6}{|c|}{ ELISA $^{a}$} & \multirow{2}{*}{$\frac{\text { IFAT }^{b}}{170}$} \\
\hline & $0^{c}$ & 30 & 60 & 105 & 150 & 170 & \\
\hline $277 * 543$ & 0 & 0 & 1 & 5 & 1 & 10 & 40 \\
\hline $096 * 060$ & 0 & 0 & 1 & 5 & 0 & 10 & 40 \\
\hline $347 * 512$ & 0 & 0 & 1 & 1 & 0 & 10 & 40 \\
\hline $318 * 026$ & 0 & 0.1 & 1 & 1 & 0 & 10 & 40 \\
\hline $584 * 533$ & 0 & 0 & 0.1 & 1 & 0 & 10 & neg \\
\hline $119 * 322$ & 0 & 0 & 1 & 5 & 0 & 10 & neg \\
\hline
\end{tabular}

$a$ :ELISA anti-PvTRAP antibodies titer expressed as the reciprocal of the last positive dilution $\times 10^{3} ; b$ : IFAT performed with air-dried $P$. vivax sporozoites. Titers represent the reciprocal of the serum end-point dilution; $c$ : underline numbers correspond to days of immunization.

by day 150 (Table II). However, all monkeys produced a rapid antibody boost $\left(1 \times 10^{4}\right)$ after inoculation with PvTRAP formulated in Freund's adjuvant on day 150. At these anti-peptide titers, parasites were recognized in IFAT assays at dilutions up to 1:400 (Table II).

No difference in IFN-g production by mononuclear cells stimulated with PvTRAP peptide was observed between experimental $\left(15-38 \times 10^{6}\right)$ and control $(14-45 \times$ $10^{6}$ ) groups. Cells from all animals responded to PHA stimulation $\left(26-465 \times 10^{6}\right)$ (Table III).

Protective efficacy of PvTRAP peptide - We initially intended to measure three outcomes to assess the protective efficacy of PvTRAP peptide: prepatent period, degree of parasitemia, and duration of infection, determined by thick and thin smears after parasite challenge. However, these two tests were found to be negative in all experimental and control monkeys. We therefore used nested PCR. Two of out six monkeys in the experimental group showed parasitemia by PCR, with prepatent periods of 16 and 53 days, whereas four out of six monkeys from the control group became infected, with a prepatent period of 53 days in one monkey and 16 days in the three remaining animals. 
TABLE III

Production of IFN- $\gamma$ by mononuclear cells from peripheral blood of Aotus monkeys immunized with PvTRAP peptide

\begin{tabular}{|c|c|c|c|c|c|c|c|c|c|}
\hline \multirow[t]{3}{*}{ Group } & \multirow[t]{3}{*}{ Monkey } & 0 & 30 & 90 & 105 & 0 & 30 & 90 & 105 \\
\hline & & \multicolumn{4}{|c|}{ Peptide } & \multicolumn{4}{|c|}{ PHA } \\
\hline & & \multicolumn{8}{|c|}{ Spot-forming units (millons) } \\
\hline \multirow[t]{8}{*}{ Experimental } & $277 * 543$ & 35 & 67.5 & 5 & 22.5 & 27.5 & 222.5 & 355 & 335 \\
\hline & $096 * 060$ & 15 & 65 & 38 & 17.5 & 17.5 & 165 & 290 & 475 \\
\hline & $347 * 512$ & 30 & 5 & 0 & 0 & 5 & 115 & 465 & 377.5 \\
\hline & $318 * 026$ & 5 & 33 & 48 & 0 & 35 & 142.5 & 480 & 265 \\
\hline & $584 * 533$ & 0 & 10 & 0 & 10 & 12.5 & 87.5 & 460 & 555 \\
\hline & $119 * 322$ & 20 & 50 & 35 & 40 & 80 & 130 & 380 & 372.5 \\
\hline & Average & 17.5 & 38.3 & 20.8 & 15 & 29.6 & 143.8 & 405.0 & 396.7 \\
\hline & $\mathrm{SD}$ & 14 & 27 & 21 & 15 & 26.9 & 46.5 & 75.6 & 103.2 \\
\hline \multirow[t]{8}{*}{ Control } & $352 * 009$ & 42.5 & 8 & 8 & 0 & 12.5 & 192.5 & 382.5 & 340 \\
\hline & $779 * 631$ & 35 & 60 & 8 & 38 & 12.5 & 200 & 460 & 567.5 \\
\hline & $306 * 297$ & 2.5 & 28 & 33 & 28 & 75 & 175 & 442.5 & 540 \\
\hline & $085 * 836$ & 0 & 58 & 25 & 0 & 25 & 195 & 432.5 & 477.5 \\
\hline & $115 * 890$ & 7.5 & 35 & 20 & 23 & 5 & 112.5 & 420 & 317.5 \\
\hline & $835 * 827$ & 0 & 83 & 30 & 0 & 27.5 & 182.5 & 340 & 552.5 \\
\hline & Average & 14.6 & 45.0 & 20.4 & 14.6 & 26.3 & 176.3 & 412.9 & 465.8 \\
\hline & $\mathrm{SD}$ & 19.1 & 26.8 & 10.9 & 16.7 & 25 & 33 & 44 & 111 \\
\hline
\end{tabular}

INF- $\gamma$ production by mononuclear cells from experimental monkeys immunized with synthetic peptide formulated in Montanide ISA-720 by I.M. (0, 30, and 90) and SC booster with peptide formulated in Freund's adjutants on 150 day and control monkeys immunized at same time only whit adjuvant

Fig. 2 shows the proportion of animals without infection following inoculation in experimental and control groups after challenge with $P$. vivax sporozoites, detected by PCR. Sixty-seven percent of monkeys immunized with PvTRAP peptide survived to the end of follow-up (day 69) without becoming infected. In contrast, only $33 \%$ of the control group survived to day 69 (Chisquared log-rank test: $6.31 \mathrm{gl}$; p: 0.01). The two-fold difference in the incidence of infection in experimental and control groups was not statistically significant (p: 0.24 ). All monkeys presented normal hematocrit and blood hemoglobin concentration from day 0 to day 210, the end of observation (data not shown). No differences between groups were observed.

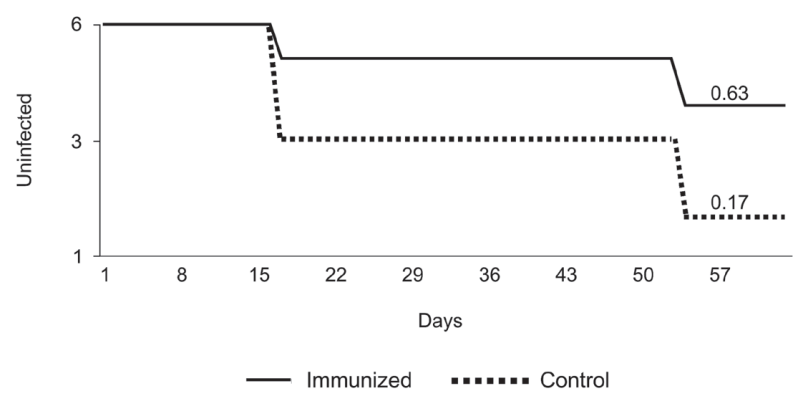

Fig. 2: proportion of animals that remain free of infection after challenge with $10^{5}$ Plasmodium vivax heterologous parasites detected by PCR of Aotus monkeys immunized with PvTRAP peptide and control monkeys immunized with adjuvant alone.

\section{DISCUSSION}

Development of a $P$. vivax malaria vaccine that blocks sporozoite invasion to the liver cell or its further intracellular multiplication would appear ideal, since this parasite species is characterized by the development of hypnozoites which are responsible for the periodic relapses induced by this parasite species. In this respect the complete protection achieved by vaccination with $P$. falciparum and $P$. vivax irradiated sporozoites and the partial protection accomplished by vaccination of human volunteers with the P. falciparum RTS-S (Alonso et al. 2004) have generated sufficient evidence for the possibility to successfully vaccinate humans against malaria.

$P$. falciparum TRAP has been considered another potential vaccine subunit. However, a Pf-TRAP based DNA vaccine (MVA ME-TRAP) could not be protected from natural infection, although apparently the vaccine was able to reduce the number of parasites that developed in the liver (Moorthy et al. 2004). No data are available about studies conducted with $P$. vivax TRAP.

In the search for $P$. vivax malaria parasite subunits with vaccine potential, here we have focused efforts towards the N-terminal region of the $P$. vivax TRAP known to contain a binding motif for invasion of liver cells. In this study we evaluated the immunogenicity and protective efficacy of a LSP containing this RII binding domain in rodents and non-human primates. The peptide proved to be immunogenic in both animal species when formulated in Freund's adjuvant not acceptable for human use, as well as in Montanide ISA 720 currently being used in human vaccine trials. Although, mice developed a steadily increasing antibody pattern since the first immunization and those antibodies cross reacted with 
the parasite in a dose dependent manner, antibodies from monkeys immunized with the peptide formulated in Montanide ISA 720 were very low. However, significant boosting was observed when the peptide formulated in Freund adjuvant was administered as well as cross reactivity with the parasite. These results indirectly confirm the good immunogenicity of the peptide provided a potent adjuvant is used, and indicate that Montanide ISA 720 is likely to be an inadequate adjuvant for inducing high levels of specific antibodies for the TRAP peptide tested; in fact several vaccine doses were required in monkeys to induce detectable antibody responses. These results are in contrast with a number of trials performed by our group where all formulations containing Montanide ISA 720 induced a rapid antibody response that usually lasted for about one year (Arévalo-Herrera et al. 2005a,b, Herrera et al. 2005). Furthermore, T cell responses appear to be the most efficient mechanisms of protection against malaria pre-erythrocytic forms; however in this study the production of IFN- $\gamma$ specifically in response to stimulation with PvTRAP peptide was not demonstrated most probably due to ineffective stimulation of $\mathrm{CD}^{+}$and/or $\mathrm{CD} 8^{+} \mathrm{T}$-cells by the vaccine formulation used. Alternatively, it is possible that production of IFN- $\gamma$ levels in Aotus by specific lymphocytes in response to PvTRAP peptide is dose-dependent and could not be detected with the immunization dose or the dose for in vitro stimulation used in this study.

Although the protection attained in this first study, was not statistically significant, the immunogenicity achieved in terms of anti-peptide antibody response that cross reacted with the parasites, together with the trend of parasitemia in control and vaccinated animals, represents an important preliminary result that deserves further studies with more potent adjuvants and an optimized sporozoite challenge system. Here we used a wild parasite strain that had not been previously adapted to grow in monkey; this may explain the difference with previous studies from our group (Jordan et al. 2005).

Finally, most probably multiple vaccine subunits would be required to achieve acceptable malaria protection. It has been proposed that the CS and TRAP, the two $P$. vivax pre-erythrocytic vaccine candidates described so far, might function synergistically by blocking sporozoite invasion, as they appear to have different antibodies binding specificities that may function at different stages of hepatocytes invasion (Sinnis \& Sim 1997). In conclusion, we believe that these preliminary results provide valuable bases for further studies to assess TRAP alone and in combination with other $P$. vivax subunit vaccine (i.e. CS, MSP-1 or others) in preclinical studies.

\section{ACKNOWLEDGMENTS}

To Liliana Soto, Miguel A Hernández, and Luz AMoreno (Malaria Vaccine and Drug Development Center) for technical support. Doug Taylor from Family Health International provided assistance in statistical analyses, Elisabeth Robinson, and Suzanne Fischer for critical reading of the manuscript.

\section{REFERENCES}

Aidoo M, Lalvani A, Allsopp CE, Plebanski M, Meisner SJ, Krausa P, Browning M, Morris-Jones S, Gotch F, Fidock DA, Ta- kiguchi M, Robson KJH, Greenwood BM, Druilhe P, Whittle HC, Hill AVS 1995. Identification of conserved antigenic components for a cytotoxic T lymphocyte-inducing vaccine against malaria. Lancet 345: 1003-1007.

Alonso PL, Sacarla IJ, Aponte JJ, Leach A, Macete E, Hilman J, Mandomando I, Spiessens B, Guinovart C, Espansa M 2004. Efficacy of the RTS, S/ASO2A vaccine against Plasmodium falciparum infection and disease in young African children ramdomised controlled trial. Lancet 364: 1411-1420.

Arévalo-Herrera M., Roggero MA, González JM, Vergara J, Corradin G, López JA and Herrera S 1998. Mapping and comparison of the B-cell epitopes recognized on the Plasmodium vivax circumsporozoite protein by immune Colombians and immunized Aotus monkeys. Ann Trop Med Parsitol 92: 539-551.

Arévalo-Herrera M, Castellanos A, Yazdany S, Shakri AR, Chitnis ChE, Dominik and Herrera S 2005a. Immunogenicity and protective efficacy of recombinant vaccine base on the receptor-binding domain of Plasmodium vivax Duffy Binding Protein in Aotus monkeys. Am J Trop Med Hyg 73: 25-31.

Arévalo-Herrera M, Solarte Y, Yasnot MF, Castellanos A, Rincon A, Saul A, Mu J, Long C, Miller L and Herrera S 2005b. Induction of transmission-blocking immunity in Aotus monkeys by vaccination with a Plasmodium vivax clinical grade PVS25 recombinant protein. Am J Trop Med Hyg 73: 32-37.

Berry G, Kitchin R, Mock P 1992. A comparison of two simple hazard ratio estimators based on the log rank test. Stat Med 11: 847-848.

Corradin G, Spertini F, Verdini A 2004. Medicinal application of long synthetic peptide technology. Expert Opin Biol Ther 4: 1629-39.

Frevert U, Sinnis P, Cerami C, Shreffler W, Takacs B, Nussenzweig V 1993. Malaria circumsporozoite protein binds to heparan sulfate proteoglycans associated with the surface membrane of hepatocytes. J Exp Med 177: 1287-1298.

Herrera S, Bonelo A, Perlaza BL, Fernández OL, Victoria L, Lenis AM, Soto L, Hurtado H, Acuña LM, Vélez JD, Palacios R, Chen-Mok M, Corradin G, Arévalo-Herrera M 2005. Safety and elicitation of humoral and cellular responses in Colombian malaria-naive volunters by a Plasmodium vivax circumsporozoite protein-derivated synthetic vaccine. Am J Trop Med Hyg 73: 3-9.

Herrera S, Corradin G, Arévalo-Herrera Myriam 2007. An update on the search for a Plasmodium vivax vaccine. Trends Parasitol 23: 122-128.

Herrera S, de Plata C, González M, Perlaza BL, Bettens F, Corradin G, Arevalo- Herrera M 1997. Antigenicity and immunogenicity of multiple antigen peptides (MAP) containing $P$. vivax CS epítopes in Aotus monkeys. Parasite Immunol 19: 161-170.

Hoffman SL, Franke E, Hollingdale M, Druilhe P 1996. In SL Hoffman, Malaria Vaccine Development: A Multi-Immune Response Approach, AMS Press, Washington DC, p. 35-75.

Jordan-Villegas A, Zapata JC, Bonelo A, Quintero G, Solarte Y, Arévalo-Herrera M, Herrera S 2005. Aotus lemurinus griseimembra monkeys: a suitable model for Plasmodium vivax sporozoite infection Am J Trop Med Hyg 73: 10-15.

Moorthy VS, Imoukhuede EB, Milligan P, Bojang K, Keating S, Kaye P, Pinder M, Gilber SC, Walraven G, Greenwood BM, 
Hill AVS 2004. A randomised, double-blind, controlled vaccine efficacy trial of DNA/MVA ME-TRAP against malaria infection in gambian adults. PLoS Med 1: 128-135.

Muller H, Reckmann I, Hollingdale MR, Bujard H, Robson KJ, Crisanti A 1993. Thrombospondin related anonymous protein (TRAP) of Plasmodium falciparum binds specifically to sulfated glycoconjugates and to HepG2 hepatoma cells suggesting a role for this molecule in sporozoite invasion of hepatocytes. EMBO J 12: 2881-2889.

Snounou G, Viriyakosol S, Zhu XP, Jarra W, Pinheiro L, do Rosario VE, Thaithong S, Brown KN 1993. High sensitivity of detection of human malaria parasite by the use of nested polymerase chain reaction. Mol Biochem Parasitol 61: 315-320.

Sinnis P, Sim BK 1997. Cell invasion by the vertebrate stages of Plasmodium. Trends Microbiol 5: 52-58.
Templeton TJ, Kaslow DC 1997. Cloning and cross-species comparison of the thrombospondin-related anonymous protein (TRAP) gene from Plasmodium knowlesi, Plasmodium vivax and Plasmodium gallinaceum. Mol Bioch Parasitol 84: $13-24$

Wizel B, Houghten R, Church P, Tine JA, Lanar DE, Gordon DM, Balou WR, Sette A, Hoffman SL 1995. HLA-A2-restricted cytotoxic T lymphocyte response to multiple Plasmodium falciparum sporozoite surface protein 2 epitopes in sporozoite-imunized volunteers. J Immunol 155: 766-775.

WHO-World Health Organization 1991. Basic laboratory methods in medical parasitology, Geneva.

Yuda M, Ishino T 2004. Liver invasion by malarial parasites how do malarial parasites break through the host barrier? Cell Microbiol 6: 1119-1125. 